\title{
Inhibition of apo(a) synthesis by several cell-growth regulating agents in two human hepatocarcinoma cell lines, SK-HEP-1 and Hep 3B cells
}

\author{
Kyong-Ja Hong ${ }^{1,2}$ and Soo-A Kim ${ }^{1}$ \\ 1 Department of Biochemistry, Catholic University Medical College, \\ Seoul 137-701, Korea \\ 2 Corresponding author.
}

Accepted 5 December 1996

Abbreviations: apo(a), apolipoprotein(a); LL-6, interleukin-6; Lp(a), lipoprotein(a); PMA, phorbol 12-myristate 13-acetate

\begin{abstract}
The effects of cell-growth regulating agents of apolipoprotein(a) [apo(a)] synthesis were examined in human hepatocarcinoma cell lines. Among four cell lines, apo(a) can be detected in three cell lines, SK-HEP-1, Hep 3B and Chang cells, but can not be detected in Hep $\mathbf{G} 2$ cells at the same protein concentration. Particularly, SK-HEP-1 cells synthesized the highest level among the examined cell lines. Two cell lines, SK-HEP-1 and Hep 3B cells were treated with several cell-growth regulating agents; dimethyl sulfoxide, all-trans retinoic acid, interleukin-6, phorbol 12-myristate 13acetate (PMA) and sodium butyrate. Culture media were harvested to determine the apo(a) level which was produced from these cells. Dimethyl sulfoxide and retinoic acid inhibited apo(a) synthesis dosedependently. In both cell lines, maximum inhibitions induced by dimethyl sulfoxide and retinoic acid were observed at $2.0 \%$ and $2.0 \mu \mathrm{M}$, respectively. PMA increased apo(a) synthesis in both cell lines. Interleukin-6 suppressed apo(a) synthesis in dose-dependent manner and completely blocked at $2,000 \mathrm{U} / \mathrm{ml}$ in Hep 3B cells, while there was no changes in SK-HEP-1 cells.
\end{abstract}

Keywords: apolipoprotein(a), lipoprotein(a)

\section{Introduction}

Lipoprotein(a) $[\mathrm{Lp}(\mathrm{a})]$ is composed of a low density lipoprotein (LDL) particle and a characteristic protein component called apolipoprotein(a) [apo(a)] which is linked to apolipoprotein B-100 (apo B-100) by disulfide bond. Based on the sequencing of apo(a) protein and cDNA, the extensive homology between apo(a) and plasminogen suggests that a high apo(a) level is a risk factor for atherosclerosis, leading to an inhibition of functions and generation of plasmin (Gaubatz et al., 1983; Utermann and Weber, 1983; McLean et al., 1987). Moreover, Lp(a) level in plasma has been reported as the best predict of coronary artery reocclusion after balloon angioplasty (Hearn et al., 1992; Solymoss et al., 1993). Unlike most other risk factors, $\mathrm{Lp}(\mathrm{a})$ level in plasma is not affected by many physiological, pharmacological or environmental influences. However, synthesis of $L p(a)$ is strictly controlled genetically (Boerwinkle et al., 1992). Under normal conditions, its level in plasma is greatly variable over a wide range among individuals, but is remarkably stable in any given individual (Boerwinkle et al., 1992).

Although the synthesis and metabolism of apo(a) is not clear, the liver is considered to be the major site of apo(a) synthesis (Kraft et al., 1989; Rainwater and Lanford, 1989; Tomlinson et al., 1989). To date, no agents have been reported to reduce the apo(a) synthesis effectively. Except niacin, tested drugs have failed to lower $L p(a)$ concentrations in plasma (Gurakar et al., 1985).

Since $L p(a)$ is detected only in a few mammalian species, primates have been the only model used to study its synthesis (Rainwater and Lanford, 1989; Tomlinson et al., 1989). Recently, atherogenesis was induced by free apo(a) and strongly enhanced by assembly of apo(a) with apo B-100 in the transgenic mice (Lawn et al., 1992; Callow et al., 1995). However, there was no studies on the regulation of apo(a) synthesis in vivo. Fortunately, most human hepatocarcinoma cell lines can normally synthesize and secrete the major plasma proteins; it has been well demonstrated that some human hepatocarcinoma cell lines could be useful models to investigate lipoprotein synthesis (Knowles et al., 1980; Javitt, 1990; Mackiewicz et al., 1991; Hahn et al., 1992).

$L p(a)$ level in plasma is elevated in cancer patients and $L p(a)$ stimulates the proliferation of human smooth muscle cells (Wright et al., 1989; Grainger et al., 1993). Previous studies have demonstrated that several cytokines and sodium butyrate can regulate the synthesis of apolipoprotein A-I (apo A-I) and apo B-100 (Kaptein et al., 1991; Ettinger et al., 1994).

In this study, to determine whether cell-growth regulating agents affect the rate of apo(a) synthesis, several cell-growth regulating agents were tested in two 
human hepatocarcinoma cell lines, SK-HEP-1 and Hep 3B cells.

\section{Materials and Methods}

\section{Materials}

Dimethyl sulfoxide, all-trans retinoic acid, sodium butyrate and phorbol 12-myristate 13-acetate (PMA) were purchased from Sigma Co. (St. Louis, MO). Fetal bovine serum was obtained from Hyclone Laboratories (Logan, UT), and nitrocellulose membrane was obtained from Schleicher \& Schuell (Germany). Mouse monoclonal antibody to human $L p(a)$ was purchased from Boehringer Mannheim Biochemica (Germany) and rabbit polyclonal antibody to human $L p(a)$ was purchased from DAKO (Denmark).

\section{Cell lines and culture}

Human hepatocarcinoma SK-HEP-1 (ATCC HTB 52), Hep G2 (ATCC HB 8065), Hep 3B (ATCC HB 8064) cells and human liver Chang (ATCC CCL 13) cells were obtained from American Type Culture Collection (Rockville, MD). These cell lines were maintained in RPMI 1640 medium containing $20 \mathrm{mM}$ Hepes, $50 \mu \mathrm{g} / \mathrm{ml}$ gentamycin, and $10 \%$ heat-inactivated fetal bovine serum. Cells were plated at an initial density of $3 \times 10^{5} / \mathrm{ml}$. Experiments were carried out with a confluent monolayer of cells 2 days after plating.

\section{Cell stimulation, viability and protein assay}

The confluent monolayer of cells was washed twice with PBS $(\mathrm{pH} 7.2)$ and incubated at $37^{\circ} \mathrm{C}$ with serumfree RPMI 1640 in the presence or absence of various agents. After 2 days, culture supernatants were harvested and concentrated to determine the apo(a) synthesis. Cell viability was determined by staining the cells with 3-(4,5-dimethyl-thiazol-2-yl)-2,5-diphenyl tetrazolium bromide (MTT) (Mosmann, 1983). Total protein concentration of culture supernatants was measured with Coomassie protein assay reagent (Pierce, Rockford, IL) using bovine serum albumin as a standard (Bradford, 1976).

\section{Determination of apo(a) synthesis}

For Western blot analysis, concentrated supernatants were separated by 5-15\% gradient SDS-PAGE as described by Laemmli (1970) and then electroblotted onto nitrocellulose membrane. After blocking with $5 \%$ non-fat milk in Tris buffered saline, $\mathrm{pH} 7.5$, membrane was incubated with mouse monoclonal antibody to human $L p(a)$ for $1.5 \mathrm{~h}$ at room temperature. Apo(a) was visualized by enhanced chemiluminescence system (Amersham, Buckinghamshire, U.K.) using anti-mouse
IgG peroxidase conjugate. The amount of apo(a) was measured by densitometry (Quick Scan, Helena, Beaumont, TX). Apo(a) was also identified by rabbit polyclonal antibody to human $L p(a)$ as described above.

\section{Results and Discussion}

\section{Demonstration of apo(a) synthesis in several human hepatocarcinoma cell lines}

To test whether available hepatocarcinoma cell lines can produce apo(a), the level of apo(a) in the culture media was determined using monoclonal antibody to human $L p(a)$. As shown in Figure 1, apo(a) but detected in SK-HEP-1, Hep 3B, and Chang cells, was not detected in Hep $\mathrm{G} 2$ cells at the same protein concentration. Especially, SK-HEP-1 cells produced a high level of apo(a). Apo(a) can also be identified by polyclonal antibody to human $L p(a)$ (data not shown). The molecular size of apo(a) synthesized in these cells was calculated to be approximately $180 \mathrm{kDa}$ under reducing condition from its electrophoretic mobility (Figure 1).

Apo(a) migrated to a position of approximately 650 kDa by SDS-PAGE in the absence of 2-mercaptoethanol (Figure 2A and 2B). In addition to $650 \mathrm{kDa}$ form, $180 \mathrm{kDa}$ protein was also detected in partial reducing condition of $0.02 \%$ 2-mercaptoethanol (Figure 2B). Based on the molecular size of apo B-100 of about $500 \mathrm{kDa}$, the difference in electrophoretic mobility between reduced and non-reduced apo(a) shows clear

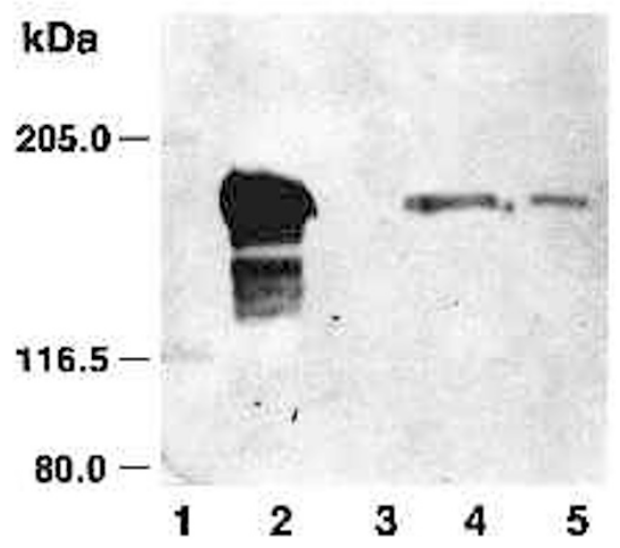

Figure 1. Expression of apo(a) in hepatoma cell lines. Cells were plated at an initial density of $3 \times 10^{5} / \mathrm{ml}$ on a 6 -well culture plate. After 2 days, media were exchanged with serum-free RPMI 1640 and cultured for 2 days. Culture supernatants $(25 \mu \mathrm{g}$ protein/lane) were analyzed by Western blotting. The positions of molecular weight standards are shown on the left. Lane 1, size marker; lane 2, SK-HEP-1; lane 3, Hep G2; lane 4, Hep 3B; lane 5, Chang cell. 

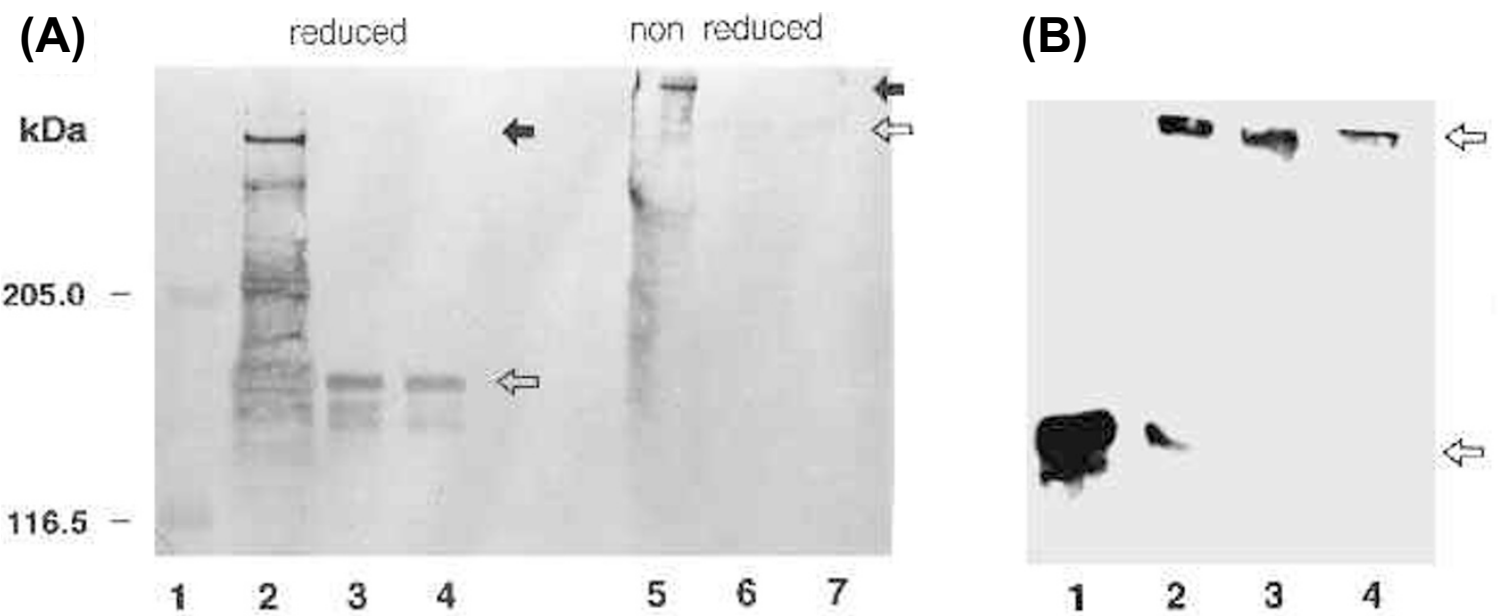

Figure 2. Electrophoretic patterns of apo(a) in reducing or non-reducing condition. (A) $\mathrm{Lp}(\mathrm{a})$ and apo(a) in normal human serum (closed arrow) and cell culture supernatants (open arrow) were analyzed by Western blotting with or without 2-mercaptoethanol. Lane 1, size marker; lanes 2 and 5 , normal human serum; lanes 3 and 6 , cell culture supernatant; lanes 4 and 7 , dimethyl sulfoxide (1.0\%) treated cell culture supernatant.

correlation with changes caused by apo B-100 binding. These results explain that the migration rate of the nonreduced form of apo(a) was extremely retarded by apo $\mathrm{B}-100$ binding because $180 \mathrm{kDa}$ of apo(a) was bound to apo B-100 and forms $650 \mathrm{kDa}$ complex. In parallel experiments, apo(a) from SK-HEP-1 cells treated with tunicamycin showed identical size to that of the untreated cells (data not shown). Apo(a) in normal human serum can be detected at normal size with the same monoclonal antibody. Moreover, purchased monoclonal antibody to $L p(a)$ had been prepared to be highly specific to human apo(a) and to be not reactive to plasminogen.

According to our results, it was suggested that 180 $\mathrm{kDa}$ component is the apo(a) expressed in human hepatocarcinoma cells, which was derived from a minimum number of kringle 4-repeats and lower glycosylation. For further studies SK-HEP-1 and Hep $3 \mathrm{~B}$ cells were used here because these cells produced large amounts of apo(a) compared with other cells.

\section{Effects of cell-growth regulating agents on apo(a) synthesis in SK-HEP-1 and Hep 3B cells}

(B) Cell culture supernatants were analyzed by Western blotting with various concentrations of 2-mercaptoethanol. Lanes 1-4: 0.2, 0.02, 0.002, $0 \% 2$ mercaptoethanol, respectively.

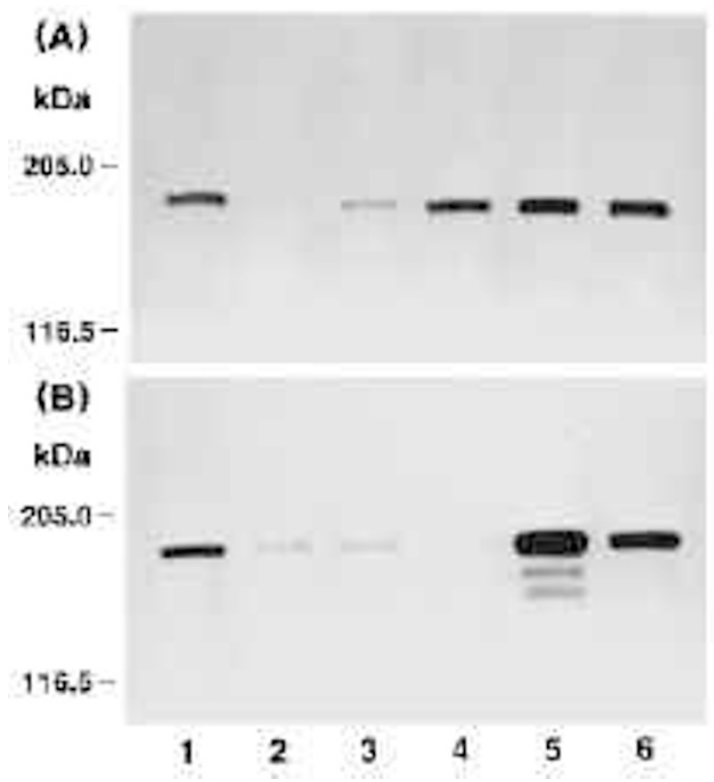

Figure 3. Effects of various cell-growth regulating agents on apo(a) synthesis in SKHEP-1 (A) and Hep 3B (B) cells. The confluent monolayer of cells was treated with indicated reagents for $48 \mathrm{~h}$ and the apo(a) which was expressed by the same cell number was analyzed by Western blotting. Lane 1, untreated control; lane 2, dimethyl sulfoxide (2.0\%); lane 3, retinoic acid $(2.0 \mu \mathrm{M})$; lane 4 , IL-6 (2,000 U/ml); lane 5 , PMA (100 nM); lane 6 , sodium butyrate $(2.0 \mathrm{mM})$. control the apo(a) synthesis in liver cells, culture media were harvested from SK-HEP-1 and Hep 3B cells treated with several agents and analyzed by Western blot. The level of apo(a) synthesized in these cells was not changed significantly depending on the time period and it was stably synthesized for 2 days (data not shown). In both cell lines, dimethyl sulfoxide and retinoic acid markedly inhibited the apo(a) synthesis while PMA elevated its synthesis (Figure 3 ). Interes-

tingly, IL-6 did not alter the apo(a) synthesis in SKHEP-1 cells, but completely blocked it in Hep 3B cells (Figure 3). All agents used here induced no significant changes in cell viability (data not shown). Total protein level secreted in culture media was not changed with 
agents except IL-6 (data not shown). IL-6 increased total protein 8 fold in SK-HEP-1 cells and 2.5 fold in Hep 3B cells compared to the control level. Hence, to exclude the effects caused by increasing the total amount of protein, the protein secreted by same cell number was loaded in a Western blot for further studies.

\section{Dose-dependent effects of dimethyl sulfoxide, retinoic acid and IL-6 on apo(a) synthesis in SK-HEP-1 and Hep 3B cells}

In two cell lines, apo(a) synthesis was dosedependently inhibited by dimethyl sulfoxide and retinoic acid (Figure 4, 5A and 5B). Remarkably, dimethyl sulfoxide and retinoic acid almost completely inhibited apo(a) synthesis at $2.0 \%$ and $2.0 \mu \mathrm{M}$, respectively (Figure 4, 5A and 5B). In Hep 3B cells, the level of secreted apo(a) was decreased depending on the concentration of IL-6 (Figure $5 \mathrm{C}$ ). Apo(a) synthesis was markedly inhibited by $1,000 \mathrm{U} / \mathrm{ml}$ of IL- 6 and almost

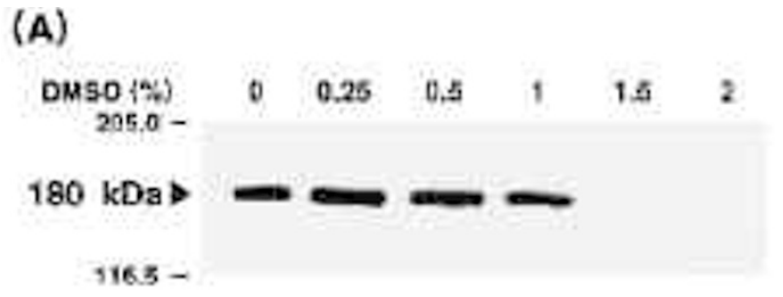

(B)

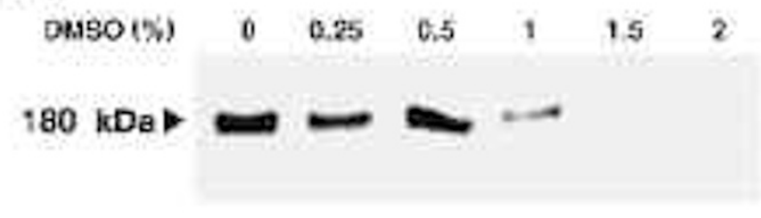

(C)

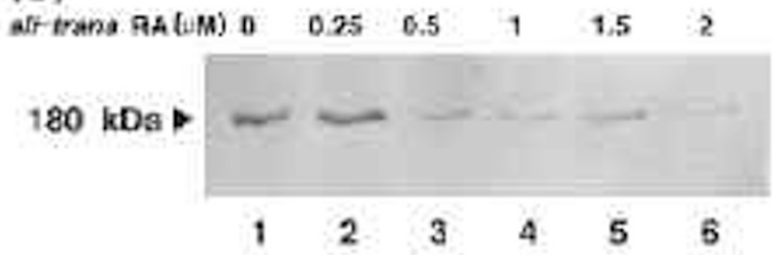

Figure 4. Dose-dependent inhibition of apo(a) synthesis by dimethyl sulfoxide ( $A$ and B) or all-trans retinoic acid (C) in SK-HEP-1 cells. (A) Cells were incubated with indicated concentrations of dimethyl sulfoxide (DMSO) for $48 \mathrm{~h}$ and culture supernatants were analyzed by Western blotting. (B) To determine the total apo(a) synthesized by SK-HEP-1 cells, cells were incubated with indicated concentrations of dimethyl sulfoxide (DMSO). After $48 \mathrm{~h}$, cell lysates and culture supernatants were mixed and analyzed by Western blotting. (C) Cells were incubated with indicated concentrations of retinoic acid (all-trans RA) for $48 \mathrm{~h}$ and the culture supernatants were analyzed by Western blotting.

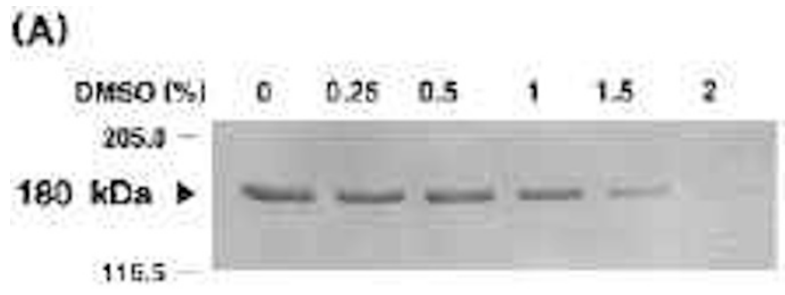

(B)

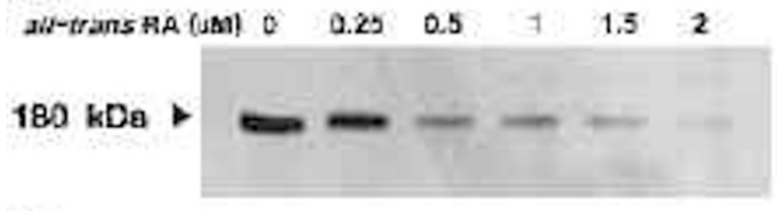

(C)

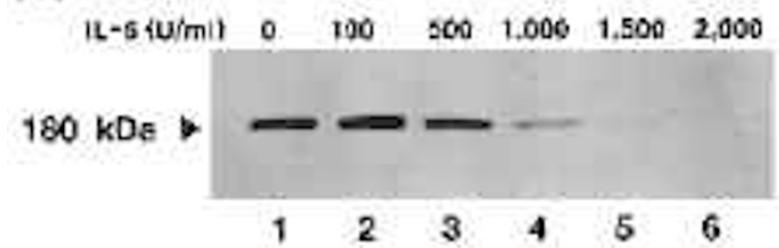

Figure. 5. Dose-dependent inhibition of apo(a) synthesis by dimethyl sulfoxide (DMSO), retinoic acid (all-trans RA) or IL-6 in Hep 3B cells. Confluent monolayer of cells were incubated with indicated concentrations of reagent for $48 \mathrm{~h}$ and the culture supernatants were analyzed by Western blotting.

completely blocked at $2,000 \mathrm{U} / \mathrm{ml}(98.9 \%)$. Recent reports demonstrated that the apo(a) gene has functional IL-6 responsive elements in the promoter region (Wade et al., 1993). Hence, our result suggests that apo(a) gene expression in Hep 3B cells can be regulated by interaction between $\mathrm{IL}-6$ and control site. By contrast, IL-6 did not inhibit apo(a) expression in SK-HEP-1 cells. Further studies on the apo(a) gene expression will provide more important informations to solve the regulatory mechanism which is modulated by the interaction between trans-acting factors and the cis element of apo(a) gene promoter.

Numerous studies reported that dimethyl sulfoxide, retinoic acid, sodium butyrate and PMA can regulate cell-growth in some types of cells. In this study, apo(a) synthesis was regulated by these agents without a significant change in cell number. Therefore, this suggests a possible means of lowering the apo(a) level and solving its regulatory mechanism.

\section{Acknowledgement}

This study was supported by a grant from the Basic 
Research Promotion Fund of the Ministry of Education, Korea, for 1995.

\section{References}

Boerwinkle, E., Leffert, C. C., Lin, J., Lackner, C., Chiesa, G. and Hobbs, H. H. (1992) Apolipoprotein(a) gene accounts for greater than $90 \%$ of the variation in plasma lipoprotein(a) concentrations. J. Clin. Invest. 90: 52-60

Bradford, M. M. (1976) A rapid and sensitive method for the quantitation of microgram quantities of protein utilizing the principle of protein-dye binding. Anal. Biochem. 72 : $248-254$

Callow, M. J., Verstuyft, J., Tangirala, R., Palinski, W. and Rubin, E. M. (1995) Atherogenesis in transgenic mice with human apolipoprotein B and lipoprotein(a). J. Clin. Invest. 96: 1639-1646

Ettinger, W. H., Varma, V. K., Sorci-Thomas, M., Parks, J. S., Sigmon, R. C., Smith, T. K. and Verdery, R. B. (1994) Cytokines decrease apolipoprotein accumulation in medium from Hep G2 cells. Arterioscler. Thromb. 14: 8-13

Gaubatz, J. W., Heideman, C., Gotto, A. M. Jr., Morrisett, J. D. and Dahlen, G. H. (1983) Human plasma lipoprotein(a): structural properties. J. Biol. Chem. 258: 45824589

Grainger, D. J., Kirschenlohr, H. L., Metcalfe, J. C., Weissberg, P. L., Wade, D. P. and Lawn, R. M. (1993) Proliferation of human smooth muscle cells promoted by lipoprotein(a). Science 260: 1655-1658

Gurakar, A., Hoeg, J. M., Kostner, G., Papadopoulos, N. M. and Brewer, H. B. Jr. (1985) Levels of lipoprotein Lp(a) decline with neomycin and niacin treatment. Atherosclerosis 57: 293-301

Hahn, S. E., Parkes, J. G. and Goldberg, D. M. (1992) Apolipoprotein synthesis and secretion in Hep G2 cells: effects of monensin and cycloheximide. Biochem. Cell Biol. 70: $1339-1346$

Hearn, J. A., Donohue, B. C., Ba'albaki, H., Douglas, J. S., King, S. B. 3rd., Lembo, N. J., Roubin, G. S. and Sgoutas, D. S. (1992) Usefulness of serum lipoprotein(a) as a predictor of restenosis after percutaneous transluminal coronary angioplasty. Am. J. Cardiol. 69: 736-739

Javitt, N. B. (1990) Hep G2 cells as a resource for metabolic studies: lipoprotein, cholesterol, and bile acids. FASEB J. 4: 161-168

Kaptein, A., Roodenburg, L. and Princen, H. M. G. (1991) Butyrate stimulates the secretion of apolipoprotein (apo) A-I and apo B-100 by the human hepatoma cell line Hep G2. Biochem. J. 278: 557-564

Knowles, B. B., Howe, C. C. and Aden, D. P. (1980) Human hepatocellular carcinoma cell lines secrete the major plasma proteins and hepatitis $B$ surface antigen. Science 209: 497-499
Kraft, H. G., Menzel, H. J., Hoppichler, F., Vogel, W. and Utermann, G. (1989) Changes of genetic apolipoprotein phenotypes caused by liver transplantations: implications for apolipoprotein synthesis. J. Clin. Invest. 83: 137-142

Laemmli, U. K. (1970) Cleavage of structural proteins during the assembly of the head of bacteriophage T4. Nature 227: 680-685

Lawn, R. M., Wade, D. P., Hammer, R. E., Chiesa, G., Verstuyft, J. G. and Rubin, E. M. (1992) Atherogenesis in transgenic mice expressing human apolipoprotein(a). Nature 360: $670-672$

Mackiewicz, A., Speroff, T., Ganapathi, M. K. and Kushner, I. (1991) Effects of cytokine combinations on acute phase protein production in two human hepatoma cell lines. $J$. Immunol. 146: 3032-3037

McLean, J. W., Tomlinson, J. E., Kuang, W. J., Eaton, D. L., Chen, E. Y., Fless, G. M. Scanu, A. M. and Lawn, R. M. (1987) cDNA sequence of human apolipoprotien(a) is homologous to plasminogen. Nature 330: 132-137

Mosmann, T. (1983) Rapid colorimetric assay for cellular growth and survival: application to proliferation and cytotoxicity assays. J. Immunol. Methods 65: 55-63

Rainwater, D. L. and Lanford, R. E. (1989) Production of lipoprotein(a) by primary baboon hepatocytes. Biochim. Biophs. Acta 1003: 30-35

Solymoss, B. C., Marcil, M., Wesolowska, E., Gilfix, B. M., Lesperance, J. and Campeau, L. (1993) Relation of coronary artery disease in women $<60$ years of age to the combined elevation of serum lipoprotein(a) and total cholesterol to high-density cholesterol ratio. Am. J. Cardiol. 72: 1215-1219

Tomlinson, J. E., McLean, J. W. and Lawn, R. M. (1989) Rhesus monkey apolipoprotein(a). J. Biol. Chem. 264: 5957-5965

Utermann, G. and Weber, W. (1983) Protein composition of $L p(a)$ lipoprotein from human plasma. FEBS Lett. 154: 357-361

Wade, D. P., Clarke, J. G., Lindahl, G. E., Liu, A. C., Zysow, B. R., Meer, K., Schwartz, K. and Lawn, R. M. (1993) 5' control regions of the apolipoprotein(a) gene and members of the related plasminogen gene family. Proc. Natl. Acad. Sci. U.S.A. 90 1369-1373

Wright, L. C., Sullivan, D. R., Muller, M., Dyne, M., Tattersall, M. H. and Mountford, C E. (1989) Elevated apolipoprotein(a) levels in cancer patients. Int. J. Cancer 43: 241 244 